\title{
Poisson distribution series on a general class of analytic functions
}

\author{
B. A. Frasin
}

\begin{abstract}
The main object of this paper is to find necessary and sufficient conditions for the Poisson distribution series to be in a general class of analytic functions with negative coefficients. Further, we consider an integral operator related to the Poisson distribution series to be in this class. A number of known or new results are shown to follow upon specializing the parameters involved in our main results.
\end{abstract}

\section{Introduction and definitions}

Let $\mathcal{A}$ denote the class of the normalized functions of the form

$$
f(z)=z+\sum_{n=2}^{\infty} a_{n} z^{n}
$$

which are analytic in the open unit disk $\mathbb{U}=\{z \in \mathbb{C}:|z|<1\}$. Further, let $\mathcal{T}$ be a subclass of $\mathcal{A}$ consisting of functions

$$
f(z)=z-\sum_{n=2}^{\infty}\left|a_{n}\right| z^{n}, \quad z \in \mathbb{U} .
$$

Let $p(n)=t_{3} n^{3}+t_{2} n^{2}+t_{1} n+t_{0}$ be a polynomial of degree 3 with real coefficients $t_{3}, t_{2}, t_{1}$ and $t_{0}$. Then a function $f$ of the form (2) is in $\mathbb{S}^{k}\left(t_{3}, t_{2}, t_{1}, t_{0}, \mu\right)$, if and only if

$$
\sum_{n=2}^{\infty} n^{k} p(n)\left|a_{n}\right| \leq \mu \quad\left(k \in \mathbb{N}_{0}=\mathbb{N} \cup\{0\}, \mu>0\right) .
$$

The class $\mathbb{S}^{k}\left(t_{3}, t_{2}, t_{1}, t_{0}, \mu\right)$ was introduced by the author in [11].

Received October 4, 2019.

2010 Mathematics Subject Classification. 30C45.

Key words and phrases. Analytic functions, Hadamard product, Poisson distribution series.

https://doi.org/10.12697/ACUTM.2020.24.16 
Remark 1.1. By suitably specializing the real constants $t_{3}, t_{2}, t_{1}, t_{0}, k$ and $\mu$, the class $\mathbb{S}^{k}\left(t_{3}, t_{2}, t_{1}, t_{0}, \mu\right)$ includes as its special cases various classes of analytic functions with negative coefficients that were considered, for example, in $[2-8,14,16,21-24,29,32,35,36]$.

Let $\gamma \in \mathbb{C} \backslash\{0\}, 0<\beta \leq 1,0 \leq \lambda \leq 1$, and $z \in \mathbb{U}$. A function $f$ of the form (2) is in the class $\mathcal{M}(\gamma, \lambda, \beta)$ if

$$
\left|\frac{1}{\gamma}\left(\frac{z f^{\prime}(z)+\lambda z^{2} f^{\prime \prime}(z)}{\lambda z f^{\prime}(z)+(1-\lambda) f(z)}-1\right)\right|<\beta,
$$

and in the class $R(\gamma, \lambda, \beta)$ if

$$
\left|\frac{1}{\gamma}\left(f^{\prime}(z)+\lambda z f^{\prime \prime}(z)-1\right)\right|<\beta .
$$

Altintaş et al. [5] introduced the classes $\mathcal{M}(\gamma, \lambda, \beta)$ and $R(\gamma, \lambda, \beta)$. They proved that a function $f$ of the form (2) is in the classes $\mathcal{M}(\gamma, \lambda, \beta)$ and $R(\gamma, \lambda, \beta)$ if and only if

$$
\sum_{n=2}^{\infty}(\lambda(n-1)+1)(n+\beta|\gamma|-1)\left|a_{n}\right| \leq \beta|\gamma|
$$

and

$$
\sum_{n=2}^{\infty} n(\lambda(n-1)+1)\left|a_{n}\right| \leq \beta|\gamma|,
$$

respectively. These classes are special cases of $\mathbb{S}^{k}\left(t_{3}, t_{2}, t_{1}, t_{0}, \mu\right)$, where $k=$ $0, t_{3}=0, t_{2}=\lambda, t_{1}=1-2 \lambda+\lambda \beta|\gamma|, t_{0}=(1-\lambda)(\beta|\gamma|-1), \quad \mu=\beta|\gamma|$ for the class $\mathcal{M}(\gamma, \lambda, \beta)$, and $k=1, t_{3}=t_{2}=0, t_{1}=\lambda, t_{0}=1-\lambda, \quad \mu=\beta|\gamma|$ for $R(\gamma, \lambda, \beta)$.

Let $\mathcal{P}(C, D)$ denote the class of analytic function in $\mathbb{U}$ which are of the form $(1+C w(z)) /(1+D w(z))$, where $-1<C<D \leq 1$ and $w(z)$ is an analytic function with $w(0)=0,|w(z)|<1$ in $\mathbb{U}$. Define

$$
\mathcal{S}^{*}(C, D)=\left\{f \in \mathcal{A}: \frac{z f^{\prime}(z)}{f(z)} \in \mathcal{P}(C, D)\right\}
$$

and

$$
\mathcal{K}(C, D)=\left\{f \in \mathcal{A}: z f^{\prime}(z) \in \mathcal{S}^{*}(C, D)\right\} .
$$

Shukla and Shukla [31] (see also [15]) gave the following necessary and sufficient conditions for functions $f$ of the form (2) to be in the classes $\mathcal{T}^{*}(C, D)=\mathcal{S}^{*}(C, D) \cap \mathcal{T}$ and $\mathcal{C}(C, D)=\mathcal{K}(C, D) \cap \mathcal{T}:$

$$
\sum_{n=2}^{\infty}(n(1+D)-(1+C))\left|a_{n}\right| \leq D-C
$$


and

$$
\sum_{n=2}^{\infty} n(n(1+D)-(1+C))\left|a_{n}\right| \leq D-C,
$$

respectively. These classes are also special cases of $\mathbb{S}^{k}\left(t_{3}, t_{2}, t_{1}, t_{0}, \mu\right)$, with $k=0, t_{3}=t_{2}=0, t_{1}=1+D, t_{0}=-(1+C), \mu=D-C$ for the class $\mathcal{T}^{*}(C, D)$, and with $k=1, t_{3}=t_{2}=0, t_{1}=1+D, t_{0}=-(1+C)$, $\mu=D-C$ for $\mathcal{C}(C, D)$.

For $0 \leq \alpha<1$ and $\gamma, \beta \geq 0$, let $\mathcal{W}(\alpha, \gamma, \beta)$ denote the class of functions $f \in \mathcal{A}$ such that

$$
\mathfrak{R}\left\{(1-\gamma+2 \beta) \frac{f(z)}{z}+(\gamma-2 \beta) f^{\prime}(z)+\beta z f^{\prime \prime}(z)\right\}>\alpha, \quad(z \in \mathbb{U}) .
$$

For some details about this class, see [30]. We can easily prove that a function $f$ of the form (2) is in the class $\mathcal{W} \mathcal{T}(\alpha, \gamma, \beta)=\mathcal{W}(\alpha, \gamma, \beta) \cap \mathcal{T}$ if and only if

$$
\sum_{n=2}^{\infty}[n(n-1) \beta+(\gamma-2 \beta) n+(1-\gamma+2 \beta)]\left|a_{n}\right| \leq 1-\alpha .
$$

We note that

$$
\mathbb{S}^{0}(0, \beta, \gamma-3 \beta, 1-\gamma+2 \beta, 1-\alpha)=\mathcal{W} \mathcal{T}(\alpha, \gamma, \beta) .
$$

The Poisson distribution, derived in 1837 by the French mathematician Siméon Denis Poisson, is a discrete probability distribution that is used to express the probability of observing a number of events in a given interval of time or space if these events occur with a known average rate and independently of the time since the last event.

A variable $X$ is said to be Poisson distributed if it takes the values $0,1,2, \ldots$ with probabilities $e^{-m}, m e^{-m} / 1 !, m^{2} e^{-m} / 2 !, \ldots$, respectively, where $m$ is called the parameter. Thus

$$
P(X=r)=\frac{m^{r} e^{-m}}{r !}, \quad r=0,1,2, \ldots
$$

In [28], Porwal (see also [21, 23]) introduced a power series whose coefficients are probabilities of Poisson distribution

$$
\mathcal{K}(m)(z)=z+\sum_{n=2}^{\infty} \frac{m^{n-1}}{(n-1) !} e^{-m} z^{n}, \quad z \in \mathbb{U},
$$

where $m>0$. By ratio test the radius of convergence of the above series is infinity. Also, Porwal [28] defined the series

$$
\mathcal{F}(m)(z)=2 z-\mathcal{K}(m)(z)=z-\sum_{n=2}^{\infty} \frac{m^{n-1}}{(n-1) !} e^{-m} z^{n}, \quad z \in \mathbb{U} .
$$


Using the Hadamard product, Porwal and Kumar [29] introduced a new linear operator $\mathcal{I}(m): \mathcal{A} \rightarrow \mathcal{A}$ defined by

$$
\mathcal{I}(m)(z) f=\mathcal{K}(m)(z) * f(z)=z+\sum_{n=2}^{\infty} \frac{m^{n-1}}{(n-1) !} e^{-m} a_{n} z^{n}, \quad z \in \mathbb{U},
$$

where $*$ denotes the convolution (or Hadamard product) of two series.

Motivated by several earlier results on connections between various subclasses of analytic and univalent functions by using hypergeometric functions (see, for example, $[9,18,33,34]$ ) and by the recent investigations of Porwal $[28,29,27]$, in the present paper we determine necessary and sufficient conditions for $\mathcal{F}(m)(z)$ to be in our new class $\mathbb{S}^{0}\left(t_{3}, t_{2}, t_{1}, t_{0}, \mu\right)$. Finally, we give conditions for the integral operator $\mathcal{G}(m)(z)=\int_{0}^{z} \zeta^{-1} \mathcal{F}(m)(\zeta) d \zeta$ to be in the class $\mathbb{S}^{1}\left(t_{3}, t_{2}, t_{1}, t_{0}, \mu\right)$.

\section{Necessary and sufficient conditions}

First we obtain necessary and sufficient conditions for $\mathcal{F}(m)(z)$ to be in $\mathbb{S}^{0}\left(t_{3}, t_{2}, t_{1}, t_{0}, \mu\right)$.

Theorem 2.1. If $m>0$ and $\mu>0$, then $\mathcal{F}(m)(z)$ is in $\mathbb{S}^{0}\left(t_{3}, t_{2}, t_{1}, t_{0}, \mu\right)$ if and only if

$$
t_{3} m^{3}+\left(t_{2}+6 t_{3}\right) m^{2}+\left(t_{1}+3 t_{2}+7 t_{3}\right) m+\left(t_{0}+t_{1}+t_{2}+t_{3}\right)\left(1-e^{-m}\right) \leq \mu .
$$

Proof. Since

$$
\mathcal{F}(m)(z)=z-\sum_{n=2}^{\infty} \frac{m^{n-1}}{(n-1) !} e^{-m} z^{n},
$$

according to (3) we must show that

$$
\sigma_{1}:=\sum_{n=2}^{\infty}\left(t_{3} n^{3}+t_{2} n^{2}+t_{1} n+t_{0}\right) \frac{m^{n-1}}{(n-1) !} \leq e^{m} \mu .
$$

Writing

$$
n=(n-1)+1, \quad n^{2}=(n-1)(n-2)+3(n-1)+1,
$$

and

$$
n^{3}=(n-1)(n-2)(n-3)+6(n-1)(n-2)+7(n-1)+1,
$$

we have

$$
\begin{aligned}
\sigma_{1}= & t_{3} \sum_{n=4}^{\infty} \frac{m^{n-1}}{(n-4) !}+\left(t_{2}+6 t_{3}\right) \sum_{n=3}^{\infty} \frac{m^{n-1}}{(n-3) !} \\
& +\left(t_{1}+3 t_{2}+7 t_{3}\right) \sum_{n=2}^{\infty} \frac{m^{n-1}}{(n-2) !}+\left(t_{0}+t_{1}+t_{2}+t_{3}\right) \sum_{n=2}^{\infty} \frac{m^{n-1}}{(n-1) !} .
\end{aligned}
$$


Using the facts that

$$
\sum_{n=2}^{\infty} \frac{m^{n-1}}{(n-1) !}=e^{m}-1 \text { and } \sum_{n=j}^{\infty} \frac{m^{n-1}}{(n-j) !}=m^{j-1} e^{m}, \quad j \geq 2,
$$

we immediately have

$$
\begin{aligned}
\sigma_{1}= & t_{3} m^{3} e^{m}+\left(t_{2}+6 t_{3}\right) m^{2} e^{m}+\left(t_{1}+3 t_{2}+7 t_{3}\right) m e^{m} \\
& +\left(t_{0}+t_{1}+t_{2}+t_{3}\right)\left(e^{m}-1\right) .
\end{aligned}
$$

But this last expression is bounded above by $\mu e^{m}$ if and only if (4) holds.

Theorem 2.2. If $m>0$ and $\mu>0$, then $\mathcal{F}(m)(z)$ is in $\mathbb{S}^{1}\left(t_{3}, t_{2}, t_{1}, t_{0}, \mu\right)$ if and only if

$$
\begin{aligned}
& t_{3} m^{4}+\left(10 t_{3}+t_{2}\right) m^{3}+\left(25 t_{3}+6 t_{2}+t_{1}\right) m^{2}+\left(15 t_{3}\right. \\
& \left.+7 t_{2}+3 t_{1}+t_{0}\right) m+\left(t_{0}+t_{1}+t_{2}+t_{3}\right)\left(1-e^{-m}\right) \leq \mu .
\end{aligned}
$$

Proof. In view of (3) we must show that

$$
\sum_{n=2}^{\infty} n\left(t_{3} n^{3}+t_{2} n^{2}+t_{1} n+t_{0}\right) \frac{m^{n-1}}{(n-1) !} \leq e^{m} \mu,
$$

or, equivalently,

$$
\sigma_{2}:=\sum_{n=2}^{\infty}\left(t_{3} n^{4}+t_{2} n^{3}+t_{1} n^{2}+t_{0} n\right) \frac{m^{n-1}}{(n-1) !} \leq e^{m} \mu .
$$

Using (5) and (6), and writing

$$
\begin{aligned}
n^{4}= & (n-1)(n-2)(n-3)(n-4)+10(n-1)(n-2)(n-3) \\
& +25(n-1)(n-2)+15(n-1)+1,
\end{aligned}
$$

we have

$$
\begin{aligned}
\sigma_{2}= & t_{3} \sum_{n=2}^{\infty} \frac{m^{n-1}}{(n-5) !}+\left(10 t_{3}+t_{2}\right) \sum_{n=2}^{\infty} \frac{m^{n-1}}{(n-4) !}+\left(25 t_{3}\right. \\
& \left.+6 t_{2}+t_{1}\right) \sum_{n=2}^{\infty} \frac{m^{n-1}}{(n-3) !}\left(15 t_{3}+7 t_{2}+3 t_{1}+t_{0}\right) \sum_{n=2}^{\infty} \frac{m^{n-1}}{(n-2) !} \\
& +\left(t_{3}+t_{2}+t_{1}+t_{0}\right) \sum_{n=2}^{\infty} \frac{m^{n-1}}{(n-1) !} \\
= & t_{3} m^{4} e^{m}+\left(10 t_{3}+t_{2}\right) m^{3} e^{m}+\left(25 t_{3}+6 t_{2}+t_{1}\right) m^{2} e^{m} \\
& +\left(15 t_{3}+7 t_{2}+3 t_{1}+t_{0}\right) m e^{m}+\left(t_{3}+t_{2}+t_{1}+t_{0}\right)\left(e^{m}-1\right) .
\end{aligned}
$$

Therefore, we see that the last expression is bounded above by $e^{m} \mu$ if and only if (7) is satisfied. 


\section{Inclusion properties}

A function $f \in \mathcal{A}$ is said to be in the class $\mathcal{R}^{\tau}(A, B), \tau \in \mathbb{C} \backslash\{0\},-1 \leq$ $B<A \leq 1$, if

$$
\left|\frac{f^{\prime}(z)-1}{(A-B) \tau-B\left[f^{\prime}(z)-1\right]}\right|<1, \quad z \in \mathbb{U} .
$$

This class was introduced by Dixit and Pal [10].

Lemma 3.1 (see [10]). If $f \in \mathcal{R}^{\tau}(A, B)$, then

$$
\left|a_{n}\right| \leq(A-B) \frac{|\tau|}{n}, \quad n \in \mathbb{N}-\{1\} .
$$

The result is sharp.

Making use of Lemma 3.1, we will study the action of the Poisson distribution series on the classes $\mathbb{S}^{0}\left(t_{3}, t_{2}, t_{1}, t_{0}, \mu\right)$ and $\mathbb{S}^{1}\left(t_{3}, t_{2}, t_{1}, t_{0}, \mu\right)$.

Theorem 3.2. Let $m>0, \mu>0$, and $f \in \mathcal{R}^{\tau}(A, B)$. Then $\mathcal{I}(m)(z) f$ is in $\mathbb{S}^{0}\left(t_{3}, t_{2}, t_{1}, t_{0}, \mu\right)$ if

$$
\begin{aligned}
& (A-B)|\tau|\left[t_{3} m^{2}+\left(3 t_{3}+t_{2}\right) m+\left(t_{1}+t_{2}+t_{3}\right)\left(1-e^{-m}\right)\right. \\
& \left.+\frac{t_{0}}{m}\left(1-e^{-m}-m e^{-m}\right)\right] \leq \mu .
\end{aligned}
$$

Proof. In view of (3) it suffices to show that

$$
\sigma_{3}:=\sum_{n=2}^{\infty}\left(t_{3} n^{3}+t_{2} n^{2}+t_{1} n+t_{0}\right) \frac{m^{n-1}}{(n-1) !}\left|a_{n}\right| \leq \mu e^{m} .
$$

Since $f \in \mathcal{R}^{\tau}(A, B)$, by Lemma 3.1 we have

$$
\sigma_{3} \leq(A-B)|\tau| \sum_{n=2}^{\infty}\left(t_{3} n^{2}+t_{2} n+t_{1}\right) \frac{m^{n-1}}{(n-1) !}+(A-B) c_{0}|\tau| \sum_{n=2}^{\infty} \frac{m^{n-1}}{n !} .
$$

Thus, using (5), we get

$$
\begin{aligned}
\sigma_{3} \leq & (A-B)|\tau|\left[t_{3} \sum_{n=3}^{\infty} \frac{m^{n-1}}{(n-3) !}+\left(3 t_{3}+t_{2}\right) \sum_{n=2}^{\infty} \frac{m^{n-1}}{(n-2) !}\right. \\
& \left.+\left(t_{1}+t_{2}+t_{3}\right) \sum_{n=2}^{\infty} \frac{m^{n-1}}{(n-1) !}+t_{0} \sum_{n=2}^{\infty} \frac{m^{n-1}}{n !}\right] \\
\leq & (A-B)|\tau|\left[t_{3} m^{2} e^{m}+\left(3 t_{3}+t_{2}\right) m e^{m}\right. \\
& \left.+\left(t_{1}+t_{2}+t_{3}\right)\left(e^{m}-1\right)+\frac{t_{0}}{m}\left(e^{m}-1-m\right)\right] .
\end{aligned}
$$

But this last expression is bounded above by $\mu e^{m}$ if (8) holds. 
Theorem 3.3. Let $m>0, \mu>0$, and $f \in \mathcal{R}^{\tau}(A, B)$. Then $\mathcal{I}(m)(z) f$ is in $\mathbb{S}^{1}\left(t_{3}, t_{2}, t_{1}, t_{0}, \mu\right)$ if

$$
\begin{aligned}
& (A-B)|\tau|\left[t_{3} m^{3}+\left(t_{2}+6 t_{3}\right) m^{2}+\left(t_{1}+3 t_{2}+7 t_{3}\right) m\right. \\
& \left.+\left(t_{0}+t_{1}+t_{2}+t_{3}\right)\left(1-e^{-m}\right)\right] \leq \mu .
\end{aligned}
$$

Proof. In view of (3) it suffices to show that

$$
\sigma_{4}:=\sum_{n=2}^{\infty} n\left(t_{3} n^{3}+t_{2} n^{2}+t_{1} n+t_{0}\right) \frac{m^{n-1}}{(n-1) !}\left|a_{n}\right| \leq \mu e^{m} .
$$

Since $f \in \mathcal{R}^{\tau}(A, B)$, by Lemma 3.1 we have

$$
\sigma_{4} \leq(A-B)|\tau| \sum_{n=2}^{\infty}\left(t_{3} n^{3}+t_{2} n^{2}+t_{1} n+t_{0}\right) \frac{m^{n-1}}{(n-1) !} .
$$

The remaining part of the proof is similar to that of Theorem 2.1, and so we omit the details.

\section{An integral operator}

In this section we obtain necessary and sufficient conditions for the integral operator

$$
\mathcal{G}(m)(z)=\int_{0}^{z} \frac{\mathcal{F}(m)(\zeta)}{\zeta} d \zeta
$$

to be in $\mathbb{S}^{1}\left(t_{3}, t_{2}, t_{1}, t_{0}, \mu\right)$.

Theorem 4.1. If $m>0$ and $\mu>0$, then the integral operator $\mathcal{G}(m)(z)$ is in $\mathbb{S}^{1}\left(t_{3}, t_{2}, t_{1}, t_{0}, \mu\right)$ if and only if the condition (4) is satisfied.

Proof. Since

$$
\mathcal{G}(m)(z)=z-\sum_{n=2}^{\infty} \frac{e^{-m} m^{n-1}}{n !} z^{n},
$$

in view of (3) we only need to show that $\sigma_{1} \leq \mu e^{m}$.

The remaining part of the proof is similar to that of Theorem 2.1, and so we omit the details.

The proof of the following theorem is much akin to that of Theorem 4.1, and so the details have been omitted.

Theorem 4.2. If $m>0$ and $\mu>0$, then the integral operator $\mathcal{G}(m)(z)$ is in $\mathbb{S}^{0}\left(t_{3}, t_{2}, t_{1}, t_{0}, \mu\right)$ if and only if

$$
t_{3} m^{2}+\left(3 t_{3}+t_{2}\right) m+\left(t_{1}+t_{2}+t_{3}\right)\left(1-e^{-m}\right)+\frac{t_{0}}{m}\left(1-e^{-m}-m e^{-m}\right) \leq \mu .
$$




\section{Corollaries and consequences}

In this section, we apply our main results in order to deduce each of the following new results.

Corollary 5.1. Let $m>0, \gamma \in \mathbb{C} \backslash\{0\}, 0<\beta \leq 1$, and $0 \leq \lambda \leq 1$.

(1) $\mathcal{F}(m)(z)$ is in $\mathcal{M}(\gamma, \lambda, \beta)$ if and only if

$$
e^{m}\left[\lambda m^{2}+(1+\lambda+\lambda \beta|\gamma|) m\right] \leq \beta|\gamma| .
$$

(2) $\mathcal{F}(m)(z)$ is in $R(\gamma, \lambda, \beta)$ if and only if

$$
\lambda m^{2}+4(1-\lambda) m+\left(1-e^{-m}\right) \leq \beta|\gamma| .
$$

(3) If $f \in \mathcal{R}^{\tau}(A, B)$, then $\mathcal{I}(m)(z) f$ is in $\mathcal{M}(\gamma, \lambda, \beta)$ if

$$
\begin{aligned}
& (A-B)|\tau|\left[\lambda m+(1-\lambda+\lambda \beta|\gamma|)\left(1-e^{-m}\right)\right. \\
& \left.+\frac{(1-\lambda)(\beta|\gamma|-1)}{m}\left(1-e^{-m}-m e^{-m}\right)\right] \leq \beta|\gamma| .
\end{aligned}
$$

(4) If $f \in \mathcal{R}^{\tau}(A, B)$, then $\mathcal{I}(m)(z) f$ is in $R(\gamma, \lambda, \beta)$ if

$$
(A-B)|\tau|\left[\lambda m+\left(1-e^{-m}\right)\right] \leq \beta|\gamma| .
$$

(5) The integral operator $\mathcal{G}(m)(z)$ is in $R(\gamma, \lambda, \beta)$ if and only if

$$
\lambda m+1-e^{-m} \leq \beta|\gamma| .
$$

(6) The integral operator $\mathcal{G}(m)(z)$ is in $\mathcal{M}(\gamma, \lambda, \beta)$ if and only if $\lambda m+(1-\lambda+\lambda \beta|\gamma|)\left(1-e^{-m}\right)+\frac{(1-\lambda)(\beta|\gamma|-1)}{m}\left(1-e^{-m}-m e^{-m}\right) \leq \beta|\gamma|$.

Corollary 5.2. Let $m>0$ and $-1<C<D \leq 1$.

(1) $\mathcal{F}(m)(z)$ is in $\mathcal{T}^{*}(C, D)$ if and only if

$$
e^{m}(1+D) m \leq D-C .
$$

(2) $\mathcal{F}(m)(z)$ is in $\mathcal{C}(C, D)$ if and only if

$$
e^{m}\left[(1+D) m^{2}+(3 D-C+2) m\right] \leq D-C .
$$

(3) $\mathcal{I}(m)(z) f$ is in $\mathcal{T}^{*}(C, D)$ if

$(A-B)|\tau|\left[(1+D)\left(1-e^{-m}\right)-\frac{(1+C)}{m}\left(1-e^{-m}-m e^{-m}\right)\right] \leq D-C$.

(4) For $f \in \mathcal{R}^{\tau}(A, B), \mathcal{I}(m)(z) f$ is in $\mathcal{C}(C, D)$ if

$$
(A-B)|\tau|\left[(1+D) m+(D-C)\left(1-e^{-m}\right)\right] \leq D-C .
$$

(5) The integral operator $\mathcal{G}(m)(z)$ is in $\mathcal{C}(C, D)$ if and only if condition (10) is satisfied.

(6) The integral operator $\mathcal{G}(m)(z)$ is in $\mathcal{T}^{*}(C, D)$ if and only if

$$
(1+D)\left(1-e^{-m}\right)-\frac{(1+C)}{m}\left(1-e^{-m}-m e^{-m}\right) \leq D-C .
$$


Corollary 5.3. Let $m>0,0 \leq \alpha<1$, and $\gamma, \beta \geq 0$.

(1) $\mathcal{F}(m)(z)$ is in $\mathcal{W T}(\alpha, \gamma, \beta)$ if and only if

$$
e^{m}\left[\beta m^{2}+\gamma m\right] \leq 1-\alpha .
$$

(2) For $f \in \mathcal{R}^{\tau}(A, B), \mathcal{I}(m)(z) f$ is in $\mathcal{W} \mathcal{T}(\alpha, \gamma, \beta)$ if

$(A-B)|\tau|\left[\beta m+(\gamma-2 \beta)\left(1-e^{-m}\right)+\frac{1-\gamma+2 \beta}{m}\left(1-e^{-m}-m e^{-m}\right)\right] \leq 1-\alpha$.

(3) The integral operator $\mathcal{G}(m)(z)$ is in $\mathcal{W T}(\alpha, \gamma, \beta)$ if and only if

$$
\beta m+(\gamma-2 \beta)\left(1-e^{-m}\right)+\frac{1-\gamma+2 \beta}{m}\left(1-e^{-m}-m e^{-m}\right) \leq 1-\alpha .
$$

Remark 5.4. By suitably specializing the real constants $t_{3}, t_{2}, t_{1}, t_{0}, k$ and $\mu$ in Theorems 2.1, 2.2, 3.3, and 4.1, we have the corresponding results obtained for various classes in $[1,12,13,17,20,21,23,28,29]$.

Further, our main results can lead to several additional new results by suitably specializing the real constants $t_{3}, t_{2}, t_{1}, t_{0}, k$ and $\mu$ in other subclasses of analytic functions with negative coefficients introduced and studied by several authors as stated in Remark 1.1.

Acknowledgements. The author would like to thank the referee for his helpful comments and suggestions.

\section{References}

[1] Ş. Altınkaya and S. Yalçın, Poisson distribution series for analytic univalent functions, Complex Anal. Oper. Theory 12(5) (2018), 1315-1319.

[2] O. Altintaş, A subclass of analytic functions with negative coefficients, Bull. Sci. Eng. Hacet. Univ. 19 (1990), 15-24.

[3] O. Altintaş and Y. Ertekin, A new subclass of analytic functions with negative coefficients, in: Current Topics in Analytic Function Theory, World Sci. Publ., River Edge, NJ, 1992, pp. 36-47.

[4] O. Altintas and S. Owa, On subclasses of univalent functions with negative coefficients, Pusan Kyongnam Math. J. 4 (1988), 41-56.

[5] O. Altintaş, Ö. Özkan, and H. M. Srivastava, Neighborhoods of a class of analytic functions with negative coefficients, Appl. Math. Lett, 13(3) (2000), 63-67.

[6] M. K. Aouf, R. M. El-Ashwah, and S. M. El-Deeb, Certain classes of univalent functions with negative coefficients and $n$-starlike with respect to certain points, Mat. Vesnik 62(3) (2010), 215-226.

[7] M. K. Aouf and H. M. Srivastava, Some families of starlike functions with negative coefficients, J. Math. Anal. Appl. 203 (1996), 762-790.

[8] R. Bharati, R. Parvatham, and A. Swaminathan, On subclasses of uniformly convex functions and corresponding class of starlike functions, Tamkang J. Math. 26(1) (1997), 17-32.

[9] N. E. Cho, S. Y. Woo, and S. Owa, Uniform convexity properties for hypergeometric functions, Fract. Calc. Appl. Anal. 5(3) (2002), 303-313.

[10] K. K. Dixit and S. K. Pal, On a class of univalent functions related to complex order, Indian J. Pure Appl. Math. 26(9) (1995), 889-896. 
[11] B. A. Frasin, An application of generalized Bessel functions on general class of analytic functions with negative coefficients, Appl. Math. E-Notes, in press.

[12] B. A. Frasin, On certain subclasses of analytic functions associated with Poisson distribution series, Acta Univ. Sapientiae Math. 11(1) (2019), 78-86.

[13] B. A. Frasin, Tariq Al-Hawary, and G. Murugusundaramoorthy, Uniformly spirallike properties for Poisson distribution series, submitted.

[14] B. A. Frasin, Tariq Al-Hawary, and Feras Yousef, Necessary and sufficient conditions for hypergeometric functions to be in a subclass of analytic functions, Afr. Mat. 30(1-2) (2019), 223-230.

[15] R. M. Goel and N. S. Sohi, Multivalent functions with negative coefficients, Indian J. Pure Appl. Math. 12(7) (1981), 844-853.

[16] V. P. Gupta and P. K. Jain, Certain classes of univalent functions with negative coefficients, Bull. Austral. Math. Soc. 15 (1976), 467-473.

[17] N. Magesh, S. Porwal, and C. Abirami, Starlike and convex properties for Poisson distribution series, Stud. Univ. Babeş-Bolyai Math. 63(1) (2018), 71-78.

[18] E. Merkes and B. T. Scott, Starlike hypergeometric functions, Proc. Amer. Math. Soc. 12 (1961), 885-888.

[19] M. L. Mogra, On a class of starlike functions in the unit disc I, J. Indian Math. Soc. 40 (1976), 159-161.

[20] G. Murugusundaramoorthy, Parabolic starlike and uniformly convex functions associated with Poisson distribution series, Bull. Transilv. Univ. Braşov 10(59) (2017), 91-100.

[21] G. Murugusundaramoorthy, Subclasses of starlike and convex functions involving Poisson distribution series, Afr. Mat. 28 (2017), 1357-1366.

[22] G. Murugusundaramoorthy and N. Magesh, On certain subclasses of analytic functions associated with hypergeometric functions, Appl. Math. Lett. 24 (2011), 494-500.

[23] G. Murugusundaramoorthy, K. Vijaya, and S. Porwal, Some inclusion results of certain subclass of analytic functions associated with Poisson distribution series, Hacettepe J. Math. Stat. 45(4) (2016), 1101-1107.

[24] G. Murugusundaramoorthy, K. Vijaya, and K. Uma, Subordination results for a class of analytic functions involving the Hurwitz-Lerch zeta function, Int. J. Nonlinear Sci. 10(4) (2010), 430-437.

[25] S. Owa, On certain classes of univalent functions in the unit disc, Kyungpook Math. J. 24(2) (1984), 127-136.

[26] K. S. Padmanabhan, On certain classes of starlike functions in the unit disc, J. Indian Math. Soc. 32 (1968), 89-103.

[27] S. Porwal, Mapping properties of generalized Bessel functions on some subclasses of univalent functions, An. Univ. Oradea Fasc. Mat. 20(2) (2013), 51-60.

[28] S. Porwal, An application of a Poisson distribution series on certain analytic functions, J. Complex Anal. (2014), Art. ID 984135, 3 pp.

[29] S. Porwal and M. Kumar, A unified study on starlike and convex functions associated with Poisson distribution series, Afr. Mat. 27(5) (2016), 1021-1027.

[30] C. Ramachandran L. Vanitha, Certain aspect of subordination for a class of analytic functions, Int. J. Math. Anal. 9(20) (2015), 979-984.

[31] N. Shukla and P. Shukla, Mapping properties of analytic function defined by hypergeometric function, II, Soochow J. Math. 25(1) (1999), 29-36.

[32] H. Silverman, Univalent functions with negative coefficients, Proc. Amer. Math. Soc. 51 (1975), 109-116.

[33] H. Silverman, Starlike and convexity properties for hypergeometric functions, J. Math. Anal. Appl. 172 (1993), 574-581. 
[34] H. M. Srivastava, G. Murugusundaramoorthy, and S. Sivasubramanian, Hypergeometric functions in the parabolic starlike and uniformly convex domains, Integral Transforms Spec. Funct. 18 (2007), 511-520.

[35] H. M. Srivastava, T. N. Shanmugam, C. Ramachandran, and S. Sivasubramanian, A new subclass of $k$-uniformly convex functions with negative coefficients, JIPAM. J. Inequal. Pure Appl. Math. 8(2) (2007), Article 43, 14 pp.

[36] K. G. Subramanian, T. V. Sudharsan, P. Balasubrahmanyam, and H. Silverman, Classes of uniformly starlike functions, Publ. Math. Debrecen 53(3-4) (1998), 309315 .

Faculty of Science, Department of Mathematics, Al al-Bayt University, MAFrAQ, JoRdAN

E-mail address: bafrasin@yahoo.com 